\title{
Supplementary Monitoring Activity Document
}

National Cancer Institute

\section{Source}

National Cancer Institute. Supplementary Monitoring Activity Document. NCI Thesaurus.

Code C115737.

Records containing additional monitoring activities, such as co-visits, and sponsorspecific monitoring activities. 